It might be thought unprofitatle to concentrate on a disease which has such an excellent prognosis, but it must be remembered that deaths have occurred ${ }^{6}$ and that pericardial constriction may be a late result. ${ }^{7}$ Moreover, it is clearly necessary for the future guidance and happiness of the patient to be able to distinguish between acute non-specific pericarditis and cardiac infarction, and it seems from Dr. Martin's quickly gathered collection either that this is not being done over the country as a whole or that a small epidemic of the disease was taking place in Orpington.

\section{Voluntary Help}

Fortunately the complementary impulses of charity and solfhelp have not altogether given way to limp reliance on the Welfare State. Donations are still readily given for inany research purposes and to help make the lives of crippled $\lrcorner$ nd sick people more endurable. At the same time large numbers of people are insuring against having to rely simply on what the Health Service provides if they fall ill. In the present state of the country these would seem to be trends that need encouraging.

The latest report ${ }^{1}$ of that old-established charity, the King Edward's Hospital Fund for London, shows what an immense amount it is doing for the improvement of the hospital services in the Greater London area. And, as the report says, "the Fund enjoys a sound working relationship with the Ministry of Health," as well as being able to "undertake projects which a Government department answerable to the Treasury might find embarrassing." Some of the grants made in 1965, the year covered by the report, are substantial-for instance, $£ 12,000$ each to the West Middlesex Hospital and the Brook Hospital, Woolwich, for the establishment of medical centres. An important purpose of such centres is to enable the hospital doctors and the general practitioners in the area to work together more closely. Altogether in the last three years the Fund has donated $£ 90,000$ for this purpose. Other gifts include $£ 11,000$ for a patients' social centre at Runwell (Psychiatric) Hospital in Essex, £10,000 to Bexley Hospital towards the cost of a rehabilitation centre in the form of a workshop, and $£ 10,000$ to Broadmoor Hospital towards the building of a hostel for patients' visitors-to mention only the largest schemes of this kind. Many other gifts ranging from under $£ 100$ to over $£ 5,000$ have been made to advance the welfare of patients, their relatives, and in some cases the nurses looking after them. In addition grants are commonly given to improve facilities for treatment.

In considering grants to make to hospitals in the Health Service the Fund looks for " an element of experiment when deciding between the merits of the many applications it receives," though sometimes they are given " to hasten essential improvements." And the report adds, "While the rate of building new hospitals remains slow, the demand for grants will grow as existing buildings age and deteriorate." Thus the Fund deserves all the support it can get at a time when public as well as private retrenchment is decreed. Moreover, in providing ideas and standards other than those of public utility it can act as a constant stimulus to the betterment of the Health Service.

\footnotetext{
Sixty-ninth Annual Report, 1965, 1966. King Edward's Hospital Fund for London, 14 Palace Court, London W.2.
}

\section{Diagnosis of Variola Minor}

The mild variety of smallpox, variola minor, appeared in England this year at least as early as 18 February. It is characteristic of the disease that the correct diagnosis waso first established only in April, ${ }^{1}$ by which time, as subse- $c$. quent inquiries disclosed, at least 22 people had had the $\Rightarrow$ disease or were still suffering from it. The man who fell illo on 18 February was a professional photographer, who helped to establish his eventual diagnosis by taking his own photo- $\frac{\bar{c}}{\overline{6}}$. graph while the rash was fully developed. Twenty-nine casesक of smallpox from the west Midlands and north Staffordshire were admitted to the Birmingham regional smallpox hospital ${ }^{\circ}$ between 29 April and 29 June, when it was closed, as all the $\overrightarrow{0}$ patients had been discharged and the infection had apparently $\vec{\omega}$ been brought under control. A preliminary account of thiso outbreak has already been published. ${ }^{2}$ But by this time cases? had been discovered in Monmouthshire, in Salford, Lanca shire, and in Solihull, Warwickshire, though none wasir immediately diagnosed as smallpox. In every one of these outbreaks a diagnosis of chicken-pox had to be revised afteriv an interval of days or weeks when it became clear that the infection was really smallpox. No connexion between anyo of these different outbreaks has so far been established, and $\mathrm{P}$ the sources of infection remain unknown.

Variola minor was endemic in England between 1923 ande $1934^{3}$; nearly 15,000 cases were notified in $1927 .{ }^{4}$ One reason for this was that the disease was so mild, comparablec in severity with chicken-pox, with a mortality of less than 3 per 1,000, that it was easily and often misdiagnosed. Great care will be needed to see that it does not become endemics again. Provided the diagnosis is made in good time, spreado can be effectively prevented by efficient control measures- $\frac{0}{0}$ isolation of the patient, disinfection of his home, vaccination, $\stackrel{2}{\vec{F}}$ and surveillance of contacts. ${ }^{5}$

The first and most important step in the diagnosis of smallpox is to think of it as a possibility whenever one is confronted with an unusual or atypical rash-or indeed any case of chicken-pox. Some illustrations to show characteristic features of variola minor and chicken-pox are reproduced at page 288. In the patient with a well-developed rash the 3 . diagnosis is easy. If vesicular or pustular lesions are stillio present on the seventh or eighth day of the rash as the predominant element, with scabbing only just beginning, ito can hardly be chicken-pox. But early in the course of then disease, or in the mild case with scanty rash, or in the patiento whose response to infection has been modified by vaccination, the diagnosis is often far from easy, and may be impossible on clinical grounds alone. Some pointers to the diagnosis follow.

First, the prodromal illness lasts one to six days, usually two to three. Nearly all patients with variola minor report a febrile influenza-like illness, with some or all of these? symptoms: headache, vomiting, pains in the limbs, backache, and shivering. Often all these symptoms are present, $\frac{\overrightarrow{0}}{\mathrm{D}}$ but the absence of one of them-for example, backache-does? not exclude the diagnosis of smallpox.

Secondly, the distribution of the rash should be noted.: It usually appears first on the face, sometimes on theo arms or hands. Even when it is very scanty the lesions ares more numerous on the head and limbs than on the trunks, the front of the trunk being especially spared. In smallpox, as opposed to chicken-pox, the axillae and popliteal spaces are said to be spared, but this is only relatively true, and pocks are often found in these sites, though less densely than on 
neighbouring parts. The rash is likely to be denser on areas subject to pressure-for example, under or against a ring, or under a belt or garter. Sunburnt areas are often more densely bocked than elsewhere. Lesions may indeed occur on the palms and soles in chicken-pox, but when these are seen the rash is very dense on the trunk. In smallpox the density on palms and soles is usually about the same as on forearm and shins and much greater than on the abdomen.

Thirdly, it is commonly believed that the appearance of the rash in a succession of crops, regularly seen in chicken-pox, is not a feature of smallpox. Although in variola major (except when fulminant or when modified by vaccination) the whole rash is usually out within 24 hours of its first appearance, in variola minor fresh skin lesions may continue to appear for up to six days. Hence during the first few days at least spots of different sizes may be present. ${ }^{2}$

Fourthly, the evolution of the smallpox rash from macule to vesicle to pustule to scab takes at least seven days; in chicken-pox the evolution is much more rapid. In variola minor the vesicles rarely rupture; secondary infection is rare. An unruptured lesion containing viscous, opaque, greyish fluid is unlikely to be due to chicken-pox. When fluid can be aspirated into a capillary tube from a vesicle of chicken-pox it is straw-coloured and transparent.

Difficulty in the differential diagnosis of smallpox may occur from chicken-pox, complications of vaccination, septic rashes, and rashes due to enteroviruses. The most important of these is chicken-pox. In addition to the points made above the pocks in chicken-pox are irregular in outline and variable in area ; they are superficially set in the skin, more so than in variola minor; when well matured they are flat, often with a crenate margin and oval shape; pocks in variola minor stand prominently on the surrounding skin and have circular outlines.

Of the complications of vaccination, eczema vaccinatum and the urticaria-like "allergic" rash are particularly worth mentioning. In the first there is usually a history of eczema and of vaccination, either of the patient or of a close contact. The lesions appear especially on the areas affected by eczema, commonly the face and areas of flexion, and do not follow the distribution characteristic of smallpox. When the rash has developed the patient looks and is severely ill, fever up to $103^{\circ} \mathrm{F}$. $\left(39.4^{\circ}\right.$ C.) being common; in variola minor there is little or no secondary fever. An allergic rash may appear seven to 12 days after vaccination and commonly presents as a blotchy erythema progressing rapidly to papular urticaria. Vesiculation is rare but may follow. Response to antihistamine compounds is often rapid.

Bullous impetigo and other infections of the skin may have to be distinguished. Some enterovirus infections, especially those due to Coxsackie group A viruses, such as hand, foot, and mouth disease, may present with vesicular lesions in the mouth and on the hands and feet. But the absence of prodromal symptoms and the small size and rapid evolution of the lesions usually enables the distinction to be made.

Laboratory investigation is essential in any case of suspected smallpox, both to confirm the diagnosis and to establish whether the variola is major or minor. A mixed outbreak of

\footnotetext{
1 Brit. med. F., 1966, 1, 1179.

- Gordon, C. W., et al., Lancet, 1966, 1, 1311.

- Brit. med. F., 1966, 1, 1248.

- Dixon, C. W., Smallpox, 1962, p. 211. London.

- Memorandum on the Control of Outbreaks of Smallpox, 1964. H.M.S.O.
}

both kinds together occurred in Britain in 1920, and the possible intrusion of cases of variola major into an outbreak of variola minor needs to be looked for. The specimens required by the laboratory are vesicle fluid in capillary tubes or in a syringe, or scrapings of lesions spread on a slide and allowed to dry. Dried virus remains alive for months. The more material within reason, the better. Scrum taken from convalescent patients free from crusts can be used to establish a retrospective diagnosis.

Nowadays a reliable and rapid diagnosis in nearly all cases can be given by electron microscopy. This does not distinguish between vaccinia and smallpox or between variolas major and minor. But it has the great advantage that a positive diagnosis of chicken-pox can be made even on crusts, though virus particles may be hard to find in these. Electron microscopy gave positive results in all but two of the cases investigated by this method during the first outbreak in the Midlands this year; photomicrographs of virus as seen in smallpox and chicken-pox lesions have recently been published. ${ }^{2}$ The gel diffusion test, a serological test for presence of virus antigen in lesions, is usually positive when an adequate sample of vesicle fluid has been taken, but is usually negative in the early, stages and may be negative when crusts are investigated.

The most sensitive test is to inoculate vesicle fluid or a saline suspension of the scrapings from the slide, or ground-up crusts, on to the chorio-allantoic membrane of a chick embryo. If live virus is present pocks appear within three days. If this test is negative the disease is most unlikely to be smallpox, provided the specimens have been properly taken from the patient. Vaccinia virus produces pocks on the membrane which are easily distinguishable from those of smallpox. Furthermore variola major can be distinguished from variola minor by its ability to produce pocks if eggs are incubated at $38.2^{\circ} \mathrm{C}$. Variola minor then produces no lesions. This is the only means of differentiating with certainty between the two in a single case.

Though variola minor fortunately kills but rarely, it can nevertheless be a most unpleasant disease to contract. Any effort to prevent its spread is worth while.

\section{Fluoridation of Water Supplies}

The artificial fluoridation of public water supplies, like the natural presence of fluoride in them, is known to be efficacious in reducing the incidence of dental caries. At the same time studies carried out in areas where the water supply contains natural or added fluoride have never shown any ill-health from it when the level of the fluoride is at one part per million. Many medical bodies in Great Britain (and abroad) have expressed their approval of the fluoridation of water as a public-health measure. They include the Medical Research Council, the British Dental Association, and the British Medical Association. But opposition to it is still widespread. The B.M.F. therefore invited Dr. Roy Duckworth, reader in oral medicine and oral pathology, London University, to review the scientific evidence on the safety and efficacy of fluoridation so that readers may be equipped with the facts of the subject. His article appears at page 283 this week. 


\section{Variola Minor and Chicken-pox}

(See Leading Article at page 255)

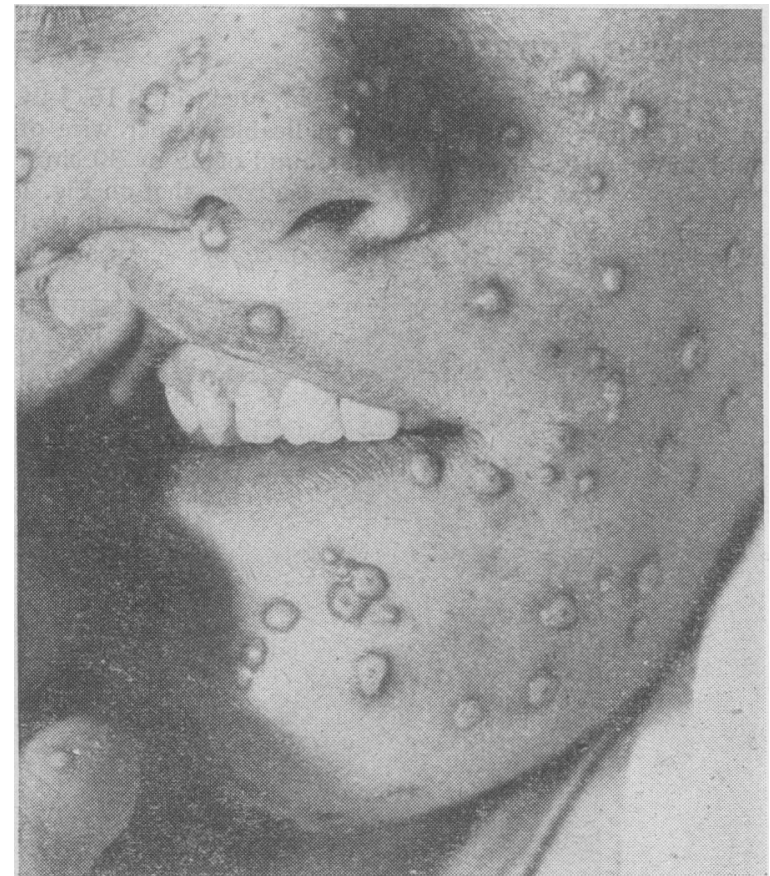

FIG. 2.-Variola minor. Late vesicular stage, becoming pustular. Note some very small papular lesions and one on the gum. Enanthematous lesions are scanty and not very common.

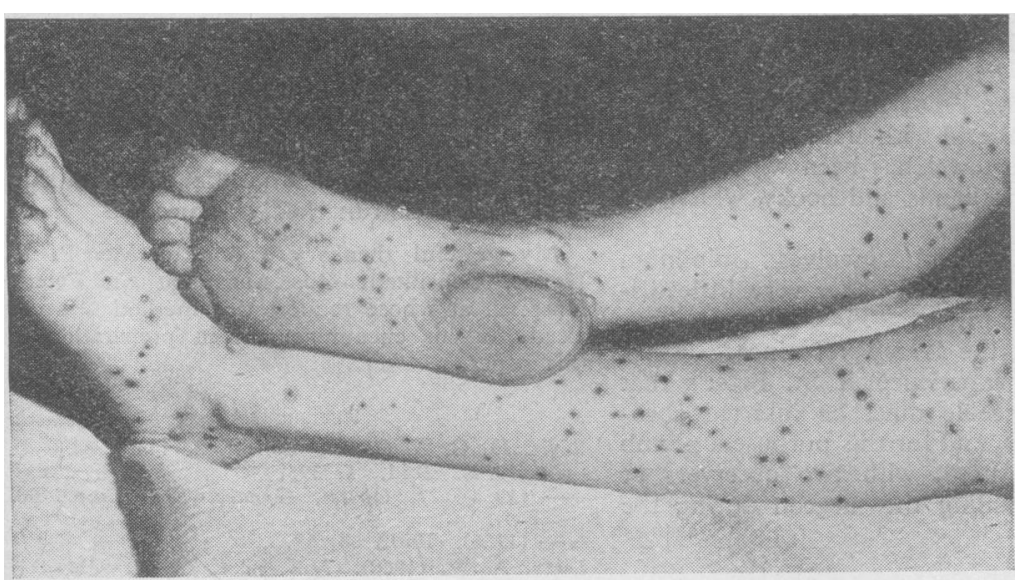

FIG. 4.- Same patient as in Fig. 1 ; the rash is now scabbing.

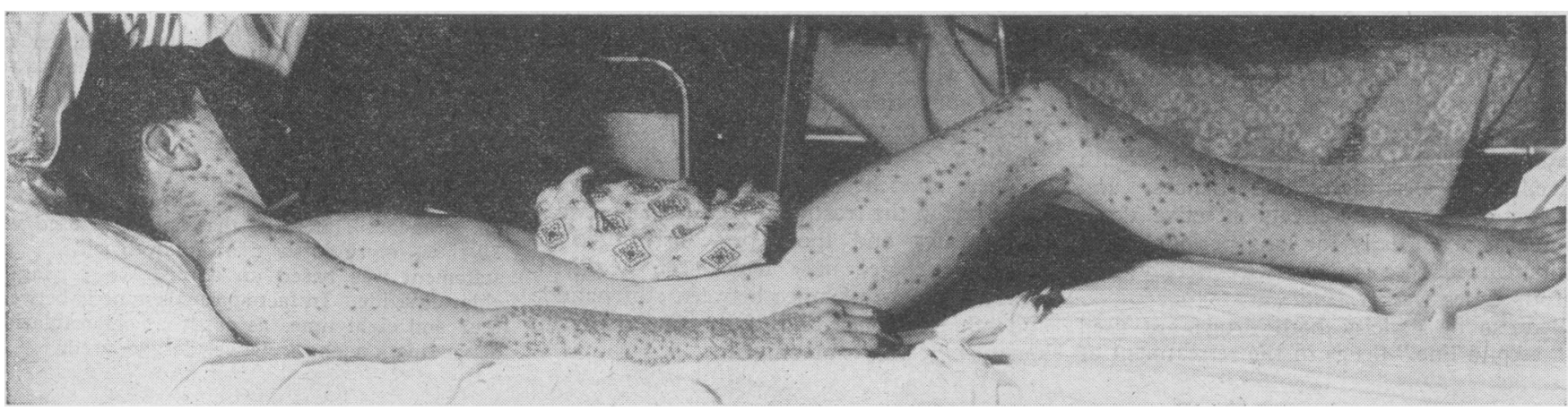

FIG. 1.-Distribution of variola minor. The rash is specially dense on forearms and neck, which had been recently sunburnt.

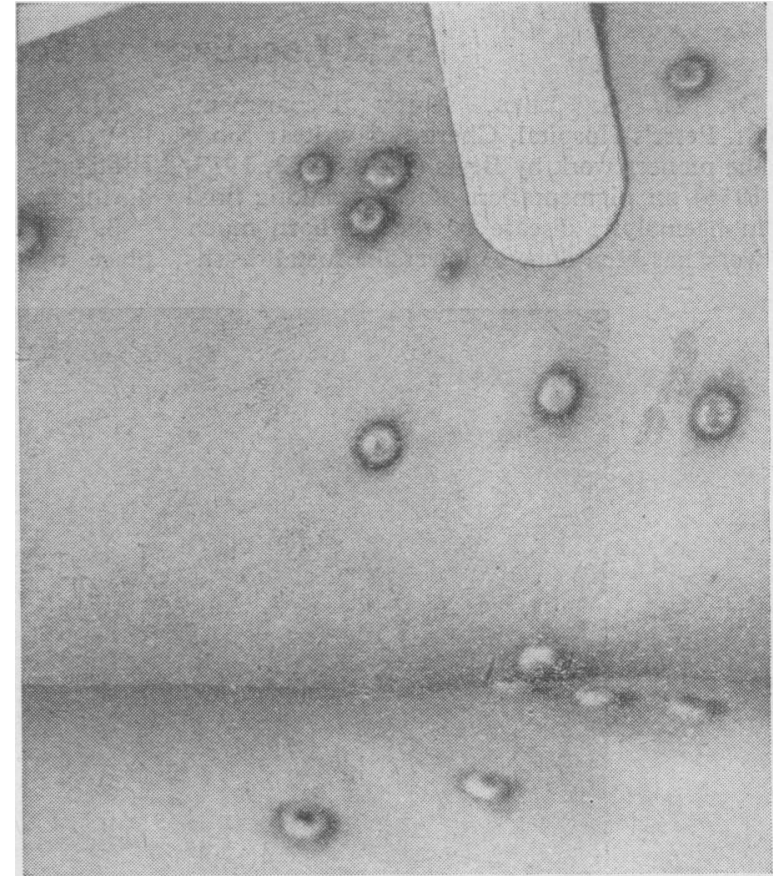

FIG. 3.-Variola minor. Eighth day of rash. Compare with

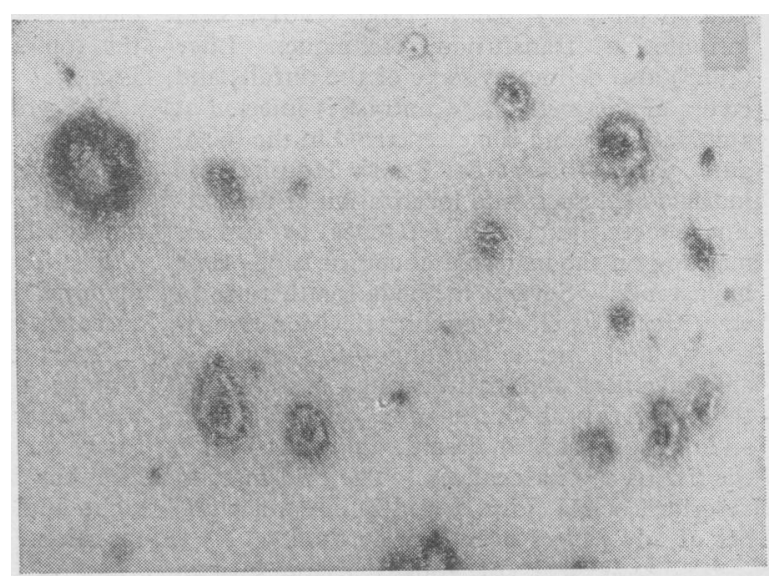

FIG. 5.-Chicken-pox lesions. Fourth or fifth day of rash. chicken-pox lesions in Fig. 5.

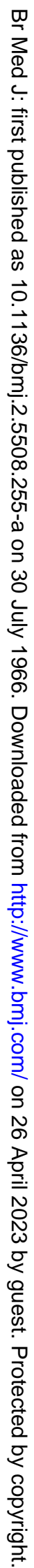

Some of these photographs have been used by the College of General Practitioners in an illustrated tape-recording. 